\title{
Diabetic Retinopathy in Southeast Asia: A Call for Ocular Telehealth Programs
}

\author{
Paolo S. Silva, 1,2,3a Jerry D. Cavallerano,,1,2 Elizabeth Paz-Pacheco, ${ }^{3 b}$ Lloyd Paul Aiello ${ }^{1,2}$ \\ ${ }^{1}$ Beetham Eye Institute, Joslin Diabetes Center, Boston, MA, USA \\ ${ }^{2}$ Department of Ophthalmology, Harvard Medical School, Boston, MA, USA \\ ${ }^{3 A}$ Departments of Ophthalmology and ${ }^{B B}$ Medicine, The Medical City, Philippines
}

\begin{abstract}
More than half-a-billion individuals are projected to have diabetes by 2030 , with the greatest increase in developing nations. Southeast Asian countries comprise a substantial proportion of this population and will need to prepare for both the rise in diabetes cases and the accompanying increase in diabetes related complications, most commonly diabetic retinopathy. Ocular telehealth programs utilizing retinal imaging for diabetic retinopathy have been established as an effective means to prevent visual loss due to diabetic retinal disease across multiple populations and in various settings. An additional value of ocular telehealth programs is their ability to integrate a multidisciplinary approach to diabetes eye care that incorporates patient education and fosters collaboration among multiple specialties. A program that provides a systematic and organized approach in delivering diabetes eye care is critically needed to successfully address this critical emerging health issue in this region of the world.
\end{abstract}

\section{Keywords: diabetes, diabetic retinopathy, ocular telehealth, telemedicine, Southeast Asia}

The estimated number of people with diabetes will likely exceed half a billion by $2030 .{ }^{1}$ This projection is higher than all prior estimates and the greatest increase will occur in developing countries. Southeast Asian countries contain a substantial proportion of this population and will need to prepare for this emerging epidemic. As has been previously observed, the rise in diabetes will be associated with an increase in the incidence of diabetic complications, including those of the kidney, nerve and, most commonly, the retina. ${ }^{2}$ In the working age population, diabetic retinal complications are one of the leading causes of visual impairment. (Figure 1) However, visual loss from diabetic retinal complications is largely preventable with early detection, careful follow-up and timely treatment. ${ }^{3}$ Early detection is critical since it allows the identification of patients at risk for visual loss and access to appropriate levels of care. An approach that has been largely espoused as a successful and cost effective means to achieve expanded and early access to diabetes eye care is the use of telemedicine. ${ }^{4}$ The American Telemedicine Association defines telemedicine as "the use of medical information exchanged from one site to another via electronic communications to improve patients' health status." 5 The closely associated term, "telehealth," is often used to encompass a broader definition of remote healthcare that does not always involve clinical services. ${ }^{5}$ Evidence based telehealth guidelines for diabetic retinopathy have been published to provide a framework for the establishment of programs in various settings. ${ }^{6}$

ISSN 0857-1074

Printed in the Philippines

Copyright (C) 2012 by the JAFES

Received September 27, 2012. Accepted October 29, 2012.
Studies across multiple countries and populations demonstrate that the prevalence of blindness and visual impairment among patients with diabetes is lowest among populations with a national program that provides retinal evaluation for all patients with diabetes. ${ }^{7-10}$ The implementation of national coverage for universal retinal evaluation of all patients with diabetes mellitus has been shown to reduce the incidence of blindness among patients with diabetes by as much as 95\%.9,11 Recent estimates in the United States have reported only 30-60\% adherence nationally with the recommended rates of retinal evaluation for diabetic retinopathy. ${ }^{12,13}$ Despite many years of widely available and promulgated guidelines recommending yearly eye examinations for patients with diabetes, ${ }^{14}$ a large proportion of patients with diabetes do not receive annual exams in the United States. $12 ; 15 ; 16$ Studies conducted in Southeast Asian have shown that the utilization of eye care services even in urban areas is staggeringly low, with an estimated $84 \%$ not receiving the minimum annual recommended eye examination. ${ }^{17}$ This rate is likely lower in rural and underserved regions where access to general medical services is a primary concern.

Diabetic retinopathy is associated with significant personal and socioeconomic burdens, but is an effectively treatable disease. Firm adherence to evidence-based standards of care is an essential and well proven approach to avoid preventable vision loss due to diabetic retinopathy.

Corresponding author: Paolo S. Silva, $M D$

Beetham Eye Institute,

Joslin Diabetes Center

One Joslin Place

Boston, Massachusetts 02215

Tel. No.: 617-309-2554

Fax No.: 617-309-2545

E-mail: paoloantonio.silva@joslin.harvard.edu 
Diabetic retinopathy is readily diagnosed by appropriate retinal examination. Retinal imaging has been an established method for determining the presence and severity of diabetic retinopathy in clinical trials for decades. ${ }^{18-21}$ Digital retinal imaging as part of an ocular telehealth program has been shown to increase surveillance and case identification rate with a proportional increase in the number of laser treatments and subsequent preservation of vision and prevention of visual loss. 22,23

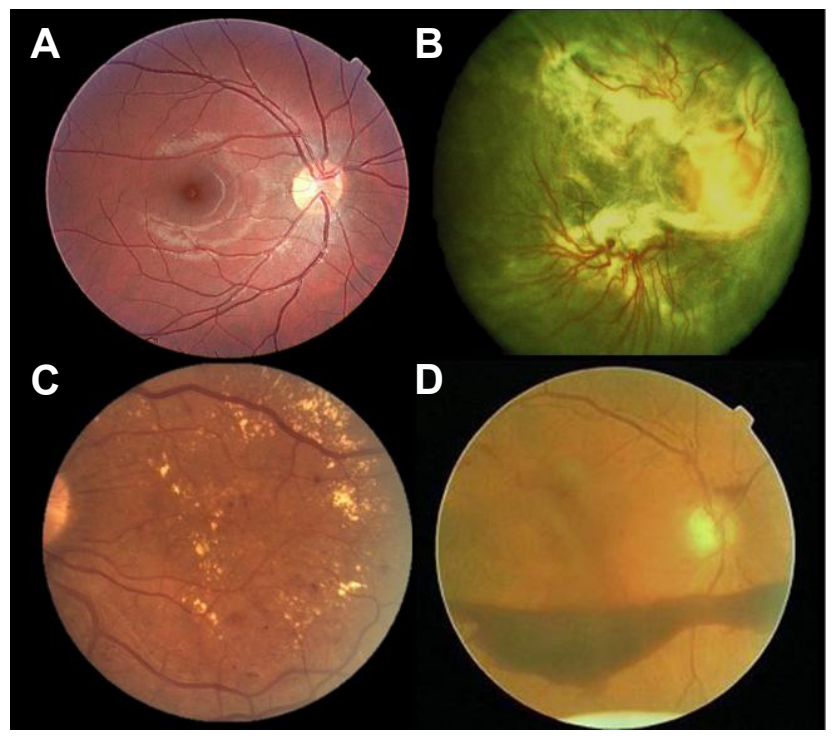

Figure 1. Retinal images of patients with diabetes. (A) Retina with no apparent diabetic retinopathy; (B) Severe traction retinal detachments; (C) Diabetic macular edema; (D) Preretinal and vitreous hemorrhage. Images B, C and $\mathrm{D}$ depict the three primary causes of visual loss due to diabetic retinal disease

The diagnosis and management of the retinal complications of diabetes begin with classification of the disease based upon the presence, location and degree of specific retinal lesions. This determination of the presence and stage of diabetic retinopathy provides the basis for increasing access to evidence-based patient care and the ability to prevent visual loss. According to the World Health Organization (WHO), telehealth programs are "designed to integrate telecommunications systems into the practice of protecting and promoting health," while telemedicine programs are "designed to integrate telecommunications into diagnostic and therapeutic intervention for the practice of curative medicine." Ocular telehealth and telemedicine programs have the potential of delivering high quality, high value, evidence-based eye care to those with no or limited access to specialty care. Such programs can also provide enhanced and more efficient care even to those with more available ocular care. Telehealth programs can also establish and enforce quality of care guidelines by linking to national clinical trial scientific data; offering education modules to healthcare professionals, patients, and communities; and facilitating recruitment for clinical trials. The American Diabetes Association recognized the value of fundus imaging in its 2012 Clinical Practice Recommendations for diabetic retinopathy, noting that "High-quality fundus photographs can detect most clinically significant diabetic retinopathy. Interpretation of images should be performed by a trained eye care provider."14 The American Diabetes Association, ${ }^{14}$ the American Academy of Ophthalmology, ${ }^{24}$ and the American Optometric Association ${ }^{25}$ generally recommend that people with diabetes mellitus undergo at minimum an annual retinal evaluation to determine the presence and severity of diabetic retinopathy and diabetic macular edema, beginning within 3-5 years of onset for type 1 diabetes ${ }^{26}$ and upon diagnosis for type 2 diabetes..$^{27,28}$ The retinal evaluation has traditionally been performed by eye doctors through dilated pupils, with or without conventional fundus photography. However, due to limited access, less than optimal use of resources, and other factors, this method has consistently failed to provide the accepted standard of care to approximately half the population with diabetes, resulting in avoidable vision loss and medical costs. Notably, this failure crosses all geographic, demographic, and socioeconomic patient categories. The development of digital retinal imaging technology has created an opportunity to increase the rate of retinal evaluation for diabetic retinopathy and has been shown to be effective and efficient across a wide range of populations and geographic locales. The technology enables the acquisition of digital retinal images in a primary care provider's (PCP) or endocrinologist's office. Images are then transmitted electronically to a remote site for evaluation and an expert interpretation. The sensitivity and specificity of digital retinal imaging performed through a validated digital retinal imaging system for detection and diagnosis of diabetic retinopathy are comparable to clinical retinal examination by eye care professionals and dilated standard fundus photography. ${ }^{29}$

${ }^{34}$ In specific and clearly defined circumstances, validated digital retinal imaging through an undilated pupil evaluated by trained eye care providers can be used in lieu of conventional fundus photography or retinal examination to document the presence and severity of diabetic retinal disease. ${ }^{30-32,35}$

\section{Ocular Telemedicine Programs in Southeast Asia}

Nearly 15 years ago, the Diabcare-Asia 1998 Study Group reported that more than half of the diabetes population in Asia is not adequately controlled. ${ }^{36}$ Subsequently, Diabcare-Asia 2003 reported that despite more effective and more available diabetes therapies, the patient outcomes remain disappointing and suboptimal. ${ }^{37}$ Correspondingly, the prevalence of diabetic microvascular complications was higher in the patients with poor control.

One of the first efforts for remote diabetic retinopathy evaluation in Southeast Asia was reported in 2000 by Ian Constable and colleagues from the Lions Eye Institute in 
Australia. ${ }^{38}$ Widespread community-based examinations were performed using hand-held portable fundus cameras in Rural Western Australia and Surabaya, Indonesia. The resolution of the imaging devices used is low as compared to current systems but this program established the value of digital imaging to extend reach for eye care services, providing a powerful tool for ophthalmology health records, teaching and research enhancing multi-specialty collaboration. This approach to reaching into the community has enormous potential for community screening for diabetic retinopathy and other blinding eye diseases. The ultimate goal of the program set forth by Dr. Constable remains unmet. An inexpensive portable imaging device that is validated in terms of sensitivity and specificity of performance and is easy to use even by local health workers, remains to be developed. With the expansion of information technology and digital imaging, the ability for image capture, image compression, image transmission, data storage and computational analysis of images has advanced substantially, potentially allowing telemedicine programs to become more cost effective. Currently, telemedicine programs for diabetic retinopathy in Asia are present in Indonesia, Thailand, Singapore, Malaysia and the Philippines. Although limited outcomes and sustainability data are available, given the diversity in the Southeast Asian region, these programs are potentially suited to address the emergence of diabetic retinal disease as the diabetic population grows.

\section{Comprehensive Diabetes Care and Education}

Although physician and patient knowledge of diabetic retinal complications has increased as a result of global informational campaigns, studies from Indonesia, Myanmar, Malaysia and Singapore have shown that patient and physician awareness regarding appropriate recommendations for care and patient awareness of the presence of retinopathy are markedly inadequate. ${ }^{17,39-41}$ Tajunisah and colleagues conducted a prospective survey to assess patient knowledge on the appropriate recommendations for diabetes eye care. ${ }^{41}$ Over $43 \%$ of patients did not know how frequently they should followup with their eye care provider and over $72 \%$ did not know what eye treatments were available. Over $80 \%$ of patients with diabetic retinopathy identified on retinal imaging were unaware of their disease. ${ }^{40}$ Across Southeast Asia, approximately $50 \%$ to as high as $80 \%$ of patients do not receive the recommended eye care. ${ }^{17,39-41}$ Even in urban areas, eye care utilization among diabetes patients remains low and it is estimated that less than $20 \%$ of patients receive the annual recommended eye examination. ${ }^{17}$

Although glycemic control and appropriate management of co-existing medical conditions remains the cornerstone of diabetes care, patient education and partnership with health care providers play an increasingly important role. Education regarding diabetic retinal complications may ameliorate the unawareness of both patients and health care providers. Addressing the emergence of diabetic retinal complications through a multidisciplinary approach that includes the patient and health care providers as partners in comprehensive diabetes care and education is critical in preserving vision and reducing the risk of vision loss from diabetes. These educational strategies may be incorporated into telemedicine programs that address both patient education and decision support for health care providers. ${ }^{42}$ Retinal imaging provides the opportunity for universal application of evidence-based diabetes eye care, focused, personal patient education, and coordination of diabetes care.

\section{Call to Action}

Given the rapidly growing diabetic population in Southeast Asia, over half of which is not achieving target glycemic goals, it is estimated that diabetic retinal complications will dramatically escalate and become the leading cause of visual loss in these regions. Southeast Asian countries are traditionally viewed as ethnically diverse and geographically dispersed. Identification and implementation of the available strategies and initiatives that may be adopted in this setting to reduce the burden of this emerging problem are greatly needed. Telemedicine efforts may provide a uniquely suited means to unite these disparate factors and enable the establishment of national programs for diabetic retinopathy surveillance and treatment, with great promise to address this critical emerging health issue in this region of the world successfully.

\section{References}

1. Whiting DR, Guariguata L, Weil C, Shaw J. IDF diabetes atlas: Global estimates of the prevalence of diabetes for 2011 and 2030. Diabetes Res Clin Pract 2011;94:311-321.

2. Self-reported visual impairment among persons with diagnosed diabetes --- United States, 1997--2010. MMWR Morb Mortal Wkly Rep 2011;60:1549-1553.

3. Mohamed Q Gillies MC, Wong TY. Management of diabetic retinopathy: A systematic review. JAMA 2007;298:902-916.

4. Rein DB, Wittenborn JS, Zhang $X$ et al. The Cost-Effectiveness of Three Screening Alternatives for People with Diabetes with No or Early Diabetic Retinopathy. Health Serv Res 2011.

5. American Telemedicine Association. Telemedicine Defined. http://www americantelemed org/i4a/pages/index cfm? pageid=3333 [serial online] 2009.

6. Li HK, Horton M, Bursell SE et al. Telehealth Practice Recommendations for Diabetic Retinopathy, Second Edition. Telemed J E Health 2011.

7. Olafsdottir E, Andersson DK, Stefansson E. Visual acuity in a population with regular screening for type 2 diabetes mellitus and eye disease. Acta Ophthalmol Scand 2007;85:40-45.

8. Fong DS, Sharza M, Chen W, Paschal JF, Ariyasu RG, Lee PP. Vision loss among diabetics in a group model Health Maintenance Organization (HMO). Am J Ophthalmol 2002;133:236-241.

9. Prasad S, Kamath GG, Jones K, Clearkin LG, Phillips RP. Prevalence of blindness and visual impairment in a population of people with diabetes. Eye (Lond) 2001;15:640-643.

10. Zoega GM, Gunnarsdottir T, Bjornsdottir S, Hreietharsson AB, Viggosson G, Stefansson E. Screening compliance and visual outcome in diabetes. Acta Ophthalmol Scand 2005;83:687-690.

11. Trautner C, Haastert B, Giani G, Berger M. Incidence of blindness in southern Germany between 1990 and 1998. Diabetologia 2001;44:147150.

12. Hartnett ME, Key IJ, Loyacano NM, Horswell RL, Desalvo KB. Perceived barriers to diabetic eye care: Qualitative study of patients and physicians. Arch Ophthalmol 2005;123:387-391. 
13. Will JC, German RR, Schuman E, Michael S, Kurth DM, Deeb L. Patient adherence to guidelines for diabetes eye care: Results from the diabetic eye disease follow-up study. Am J Public Health 1994;84:1669-1671.

14. Standards of medical care in diabetes--2012. Diabetes Care 2012;35 Suppl 1:S11-S63.

15. Thompson JW, Bost J, Ahmed F, Ingalls CE, Sennett C. The NCQA's quality compass: Evaluating managed care in the United States. Health Aff (Millwood) 1998;17:152-158.

16. Saadine JB, Fong DS, Yao J. Factors associated with follow-up eye examinations among persons with diabetes. Retina 2008;28:195-200.

17. Adriono G, Wang D, Octavianus C, Congdon N. Use of eye care services among diabetic patients in urban Indonesia. Arch Ophthalmol 2011;129:930-935.

18. Grading diabetic retinopathy from stereoscopic color fundus photographs--An extension of the modified Airlie House classification. ETDRS report number 10. Early Treatment Diabetic Retinopathy Study Research Group. Ophthalmology 1991;98:786-806.

19. The effect of intensive treatment of diabetes on the development and progression of long-term complications in insulin-dependent diabetes mellitus. The Diabetes Control and Complications Trial Research Group. N Engl J Med 1993;329:977-986.

20. Intensive blood-glucose control with sulphonylureas or insulin compared with conventional treatment and risk of complications in patients with type 2 diabetes (UKPDS 33). UK Prospective Diabetes Study (UKPDS) Group. Lancet 1998;352:837-853.

21. Aldington SJ, Kohner EM, Meuer S, Klein R, Sjolie AK. Methodology for retinal photography and assessment of diabetic retinopathy: The EURODIAB IDDM complications study. Diabetologia 1995;38:437444

22. Wilson C, Horton M, Cavallerano J, Aiello LM. Addition of primary care-based retinal imaging technology to an existing eye care professional referral program increased the rate of surveillance and treatment of diabetic retinopathy. Diabetes Care 2005;28:318-322.

23. Conlin PR, Fisch BM, Orcutt JC, Hetrick BJ, Darkins AW. Framework for a national teleretinal imaging program to screen for diabetic retinopathy in Veterans Health Administration patients. J Rehabil Res Dev 2006;43:741-748.

24. American Academy of Ophthalmology Retina Panel. Preferred Practice Guidelines. Diabetic Retinopathy. San Francisco, CA: American Academy of Ophthalmology, 2008

25. Optometric Clinical Practice Guideline: Care of the Patient with Diabetes Mellitus. [3rd Edition]. 2002. American Optometric Association. St Louis. MO.

26. Klein R, Klein BE, Moss SE, Davis MD, DeMets DL. The Wisconsin epidemiologic study of diabetic retinopathy. II. Prevalence and risk of diabetic retinopathy when age at diagnosis is less than 30 years. Arch Ophthalmol 1984;102:520-526.

27. Klein R, Klein BE, Moss SE, Davis MD, DeMets DL. The Wisconsin epidemiologic study of diabetic retinopathy. III. Prevalence and risk of diabetic retinopathy when age at diagnosis is 30 or more years. Arch Ophthalmol 1984;102:527-532.

28. Diabetes Prevention Program Research Group. The prevalence of retinopathy in impaired glucose tolerance and recent-onset diabetes in the Diabetes Prevention Program. Diabet Med 2007;24:137-144.

29. Ahmed J, Ward TP, Bursell SE, Aiello LM, Cavallerano JD, Vigersky RA. The sensitivity and specificity of nonmydriatic digital stereoscopic retinal imaging in detecting diabetic retinopathy. Diabetes Care 2006;29:2205-2209.

30. Maker MP, Noble J, Silva PS et al. Automated Retinal Imaging System (ARIS) Compared with ETDRS Protocol Color Stereoscopic Retinal Photography to Assess Level of Diabetic Retinopathy. Diabetes Technol Ther 2012.

31. Silva PS, Cavallerano JD, Sun JK, Noble J, Aiello LM, Aiello LP. Nonmydriatic Ultrawide Field Retinal Imaging Compared with Dilated Standard 7-Field 35-mm Photography and Retinal Specialist Examination for Evaluation of Diabetic Retinopathy. Am J Ophthalmol 2012

32. Silva PS, Walia S, Cavallerano JD et al. Comparison of Low-Light Nonmydriatic Digital Imaging with 35-mm ETDRS Seven-Standard Field Stereo Color Fundus Photographs and Clinical Examination. Telemed J E Health 2012;18:492-499.

33. Bursell SE, Cavallerano JD, Cavallerano AA et al. Stereo nonmydriatic digital-video color retinal imaging compared with Early Treatment Diabetic Retinopathy Study seven standard field 35- $\mathrm{mm}$ stereo color photos for determining level of diabetic retinopathy. Ophthalmology 2001;108:572-585.

34. Chow SP, Aiello LM, Cavallerano JD et al. Comparison of nonmydriatic digital retinal imaging versus dilated ophthalmic examination for nondiabetic eye disease in persons with diabetes. Ophthalmology 2006;113:833-840.

35. Cavallerano JD, Aiello LP, Cavallerano AA et al. Nonmydriatic digital imaging alternative for annual retinal examination in persons with previously documented no or mild diabetic retinopathy. Am J Ophthalmol 2005;140:667-673.

36. Chuang LM, Tsai ST, Huang BY, Tai TY. The status of diabetes control in Asia--A cross-sectional survey of 24317 patients with diabetes mellitus in 1998. Diabet Med 2002;19:978-985.

37. Mohamed M. An audit on diabetes management in Asian patients treated by specialists: the Diabcare-Asia 1998 and 2003 studies. Curr Med Res Opin 2008;24:507-514.

38. Constable IJ, Yogesan K, Eikelboom R, Barry C, Cuypers M. Fred Hollows lecture: Digital screening for eye disease. Clin Experiment Ophthalmol 2000;28:129-132.

39. Muecke JS, Newland HS, Ryan P et al. Awareness of diabetic eye disease among general practitioners and diabetic patients in Yangon, Myanmar. Clin Experiment Ophthalmol 2008;36:265-273.

40. Huang OS, Tay WT, Tai ES et al. Lack of awareness amongst community patients with diabetes and diabetic retinopathy: The Singapore Malay eye study. Ann Acad Med Singapore 2009;38:10481055.

41. Tajunisah I, Wong P, Tan L, Rokiah P, Reddy S. Awareness of eye complications and prevalence of retinopathy in the first visit to eye clinic among type 2 diabetic patients. Int J Ophthalmol 2011;4:519-524.

42. Fonda SI, Bursell SE, Lewis DG, Garren J, Hock K, Cavallerano J. The relationship of a diabetes telehealth eye care program to standard eye care and change in diabetes health outcomes. Telemed J E Health 2007;13:635-644. 